\title{
TERNARY QUARTIC APPROACH FOR POSITIVE 4TH ORDER DIFFUSION TENSORS REVISITED
}

\author{
Aurobrata Ghosh, Rachid Deriche \\ INRIA Sophia Antipolis-Méditerranée \\ Project Team Odyssée \\ Sophia Antipolis, France
}

\begin{abstract}
In Diffusion Magnetic Resonance Imaging (D-MRI), the 2nd order diffusion tensor has given rise to a widely used tool Diffusion Tensor Imaging (DTI). However, it is known that DTI is limited to a single prominent diffusion direction and is inaccurate in regions of complex fiber structures such as crossings. Various other approaches have been introduced to recover such complex tissue micro-geometries, one of which is Higher Order Cartesian Tensors. Estimating a positive diffusion function has also been emphasised mathematically, since diffusion is a physical quantity. Recently there have been efforts to estimate 4th order diffusion tensors from Diffusion Weighted Images (DWIs), which are capable of describing crossing configurations with the added property of a positive diffusion function. We take up one such, the Ternary Quartic approach, and reformulate the estimation equation to facilitate the estimation of the non-negative 4th order diffusion tensor. With our modified approach we test on synthetic, phantom and real data and confirm previous results.
\end{abstract}

Index Terms - Diffusion-MRI, Higher Order Tensors, Ternary Quartics, Diffusion Propagator

\section{INTRODUCTION}

Diffusion Magnetic Resonance Imaging (D-MRI) provides a sophisticated tool to study the connectivity of the brain's white matter in vivo. This non-invasive approach makes it possible to determine the micro-structure of the tissue by measuring and quantizing the diffusion of water molecules in a restricted environment. This allows to infer the underlying geometry. More specifically partial directional diffusion information contained in multiple Diffusion Weighted Images (DWIs) are integrated using a reconstruction model to generate an image where at every voxel a diffusion function indicates prominent diffusion directions which reflect the geometry of the tissue and point out important fiber bundles.

The earliest proposed diffusion function employing a 2 nd order Cartesian tensor has given rise to Diffusion Tensor Imaging [1], the most popularly utilised approach today. The

\author{
Maher Moakher
}

\author{
École Nationale d'Ingénieurs de Tunis (ENIT) \\ LAMSIN \\ Tunis, Tunisia
}

diffusion function was defined as $D(\mathbf{g})=\mathbf{g}^{T} \mathbf{D} \mathbf{g}$, where $\mathbf{g}$ is the diffusion weighting magnetic gradient vector and $\mathbf{D}$ is the 2nd order tensor to be estimated from a set of DWIs. It is well known, however, that the 3D, 2nd order tensor is incapable of describing more than one prominent fiber direction, and thus is inaccurate when complex fiber configurations are present such as crossings.

In [2], Higher Order Tensors (HOT) were introduced to allow for a more complex diffusion function capable of describing crossing fiber configurations. The diffusion function was remodelled to employ a 3D, Cartesian HOT and written as

$D(\mathbf{g})=\sum_{j_{1}=1}^{3} \sum_{j_{2}=1}^{3} \cdots \sum_{j_{k}=1}^{3} D_{j_{1} j_{2} \ldots j_{k}} g_{j_{1}} g_{j_{2}} \ldots g_{j_{k}}$, where $g_{j_{i}}$ are again the coordinates of the magnetic gradient vector and $D_{j_{1} j_{2} \ldots j_{k}}$ are now the independent coefficients of the k-th order diffusion tensor.

Since diffusion is a physical quantity it is meaningless if the diffusion function is negative in any direction. The authors of [3] worked with a 4th order diffusion tensor with this added constraint of non-negative diffusion. For the 4th order they rearranged the tensor indices to write the diffusion function as

$$
D(\mathbf{g})=\sum_{i+j+k=4} D_{i, j, k} g_{1}^{i} g_{2}^{j} g_{3}^{k} .
$$

In this form (1) can also be interpreted as a Ternary Quartic (TQ) in the three variables $g_{1}, g_{2}$ and $g_{3}$. The authors then used Hilbert's theorem on non-negative real TQs, proved in 1888 (see [4]), which states that a non-negative real ternary quartic is the sum of three squares of quadratic forms. The authors then parameterized the diffusion function as

$D(\mathbf{g})=\left(\mathbf{v}^{T} \mathbf{c}_{1}\right)^{2}+\left(\mathbf{v}^{T} \mathbf{c}_{2}\right)^{2}+\left(\mathbf{v}^{T} \mathbf{c}_{3}\right)^{2}=\mathbf{v}^{T} \mathbf{C} \mathbf{C}^{T} \mathbf{v}=\mathbf{v}^{T} \mathbf{G}^{T} \mathbf{v}$,

where $\mathbf{v}=\left[g_{1}^{2}, g_{2}^{2}, g_{3}^{2}, g_{1} g_{2}, g_{1} g_{3}, g_{2} g_{3}\right]$ corresponds to the gradient vector and $\mathbf{G}$ is known as the Gram matrix. The authors estimated $\mathbf{C}$ from the DWIs. This parameterization then inherently guarantees that the diffusion function is nonnegative for every direction. The 15 independent coefficients of the 4th order diffusion tensor $\mathbf{D}$ in (1) can be computed from $\mathbf{G}$ (see $[3,4])$. 
However, $\mathbf{C}$ is a $6 \times 3$ matrix and when decomposed into two blocks $\mathbf{C}=[\mathbf{A}, \mathbf{B}]^{T}$ where $\mathbf{A}$ and $\mathbf{B}$ are $3 \times 3$ matrices one observes that $\mathbf{C O}$, for any orthogonal $3 \times 3$ matrix $\mathrm{O}$ also results in the same Gram matrix, $\mathbf{C O}(\mathbf{C O})^{T}=$ $\mathbf{C C}^{T}=\mathbf{G}$. To overcome this ambiguity, the authors used a QR-decomposition of $\mathbf{A}$.

In this paper we take up again the diffusion function written as a TQ as in (1) and use the same parameterization provided by Hilbert's theorem as suggested above. But we reformulate (2) to naturally overcome the orthogonal matrix class ambiguity, and achieve the same results. We test our modified approach on synthetic, phantom and real data and confirm the importance of the non-negative diffusion constraint.

\section{METHOD}

In our approach we use the same diffusion function (1), though for the sake of simplicity we replace the three variables $g_{1}, g_{2}, g_{3}$ of the TQ by $x, y, z$. Hilbert's theorem tells us that if (1) is non-negative then it can be written as a sum of three squares of quadratic forms

$$
D(x, y, z)=\psi_{1}^{2}(x, y, z)+\psi_{2}^{2}(x, y, z)+\psi_{3}^{2}(x, y, z),
$$

where $\psi_{i}(x, y, z)=a_{i} x^{2}+b_{i} y^{2}+c_{i} z^{2}+2 \alpha_{i} x y+2 \beta_{i} x z+$ $2 \gamma_{i} y z$. Each quadratic form is known if its 6 unknown coefficients can be estimated from the DWIs. Therefore we set the unknowns to be $\mathbf{x}_{i}=\left[a_{i}, b_{i}, c_{i}, 2 \alpha_{i}, 2 \beta_{i}, 2 \gamma_{i}\right]^{T}$ for $i=1,2,3$ and define the vector $\mathbf{X}=\left[\mathbf{x}_{1}^{T}, \mathbf{x}_{2}^{T}, \mathbf{x}_{3}^{T}\right]^{T}$. Equ.3, for any gradient direction $\mathbf{g}$, can be therefore written as

$$
D\left(\mathbf{x}_{1}, \mathbf{x}_{2}, \mathbf{x}_{3}\right)=\mathbf{x}_{1}^{T} \mathbf{v} \mathbf{v}^{T} \mathbf{x}_{1}+\mathbf{x}_{2}^{T} \mathbf{v} \mathbf{v}^{T} \mathbf{x}_{2}+\mathbf{x}_{3}^{T} \mathbf{v} \mathbf{v}^{T} \mathbf{x}_{3},
$$

where $\mathbf{v}$ corresponds to the gradient direction as seen earlier, and we have rewritten the diffusion function $D$ as a function of the unknowns that need to be estimated. In other words

$$
\begin{aligned}
D\left(\mathbf{x}_{1}, \mathbf{x}_{2}, \mathbf{x}_{3}\right) & =\left[\mathbf{x}_{1}^{T}, \mathbf{x}_{2}^{T}, \mathbf{x}_{3}^{T}\right]\left[\begin{array}{ccc}
\mathbf{V} & \mathbf{0} & \mathbf{0} \\
\mathbf{0} & \mathbf{V} & \mathbf{0} \\
\mathbf{0} & \mathbf{0} & \mathbf{V}
\end{array}\right]\left[\begin{array}{l}
\mathbf{x}_{1} \\
\mathbf{x}_{2} \\
\mathbf{x}_{3}
\end{array}\right] \\
& =\mathbf{X}^{T} \mathbf{W} \mathbf{X},
\end{aligned}
$$

where $\mathbf{V}=\mathbf{v v}^{T}$.

Comparing (2) and (4) provides an insight into the simplicity of the modification which we have proposed by considering the coefficients of the quadratic forms as the unknowns. This naturally resolves the ambiguity of the orthogonal matrices in (2). What is more, by writing $\mathbf{C}^{\prime}=\left[\mathbf{x}_{1}, \mathbf{x}_{2}, \mathbf{x}_{3}\right]$ which is a $6 \times 3$ matrix, it is easy to see that the Gram matrix can be similarly computed from $\mathbf{G}=\mathbf{C}^{\prime} \mathbf{C}^{\prime T}$. Therefore, we estimate $\mathbf{X}$ from the DWIs and then shuffling the $\mathbf{x}_{i}$ s compute the Gram matrix. Once $\mathbf{G}$ is computed we then proceed as in $[3,4]$ to extract the 15 unknown coefficients of the 4th order diffusion tensor.
However, note that we are estimating totally 18 unknown coefficients of the 3 quadratic forms, whereas the symmetric 4th order diffusion tensor has only 15 independent coefficients. We surmise that this can be resolved by adding appropriate supplementary constraints to the problem and is an area of interest.

To estimate the unknown coefficients from a set of DWIs we minimize the following energy equation based on the modified and linearized Stejskal-Tanner equation

$$
E(\mathbf{X})=\frac{1}{2} \sum_{i=1}^{N}\left(\frac{1}{b} \log \left(\frac{S_{i}}{S_{0}}\right)+\mathbf{X}^{T} \mathbf{W}_{i} \mathbf{X}\right)^{2},
$$

where $N$ is the number of DWIs. Although here we have used the linearized form of the Stejskal-Tanner equation, it is equally possible to use the exponential form.

To minimize the energy function its gradient can be computed to be

$$
\nabla_{\mathbf{X}} E=\sum_{i=1}^{N}\left(\frac{1}{b} \log \left(\frac{S_{i}}{S_{0}}\right)+\mathbf{X}^{T} \mathbf{W}_{i} \mathbf{X}\right)\left(\mathbf{W}_{i}+\mathbf{W}_{i}^{T}\right) \mathbf{X} .
$$

In our implementation we have used the Broyden-FletcherGoldfarb-Shanno (BFGS) method, which is a well-known quasi-Newton optimization algorithm for non-linear problems.

\section{DIFFUSION PROPAGATOR}

The 4th order diffusion tensor estimates the ADC from the DWIs. However it is well known that the maxima of the ADC do not correspond to the dominant fiber bundle directions in regions of complex fiber configurations such as crossings [5]. The ADC is related to the diffusion weighted signal by the Stejskal-Tanner equation. From the diffusion signal it is possible to calculate the Diffusion Propagator since one is the Fourier transform of the other $P(\mathbf{r})=\int E(\mathbf{q}) \exp \left(-2 \pi i \mathbf{q}^{T} \mathbf{r}\right) d \mathbf{q}$, where $\mathbf{q}$ is the reciprocal displacement vector corresponding to the magnetic gradient vector $\mathbf{g}, E(\mathbf{q})$ is the signal value associated with the vector $\mathbf{q}$ divided by the zero gradient signal, and $\mathbf{r}$ is the spin displacement vector. When the diffusion propagator is evaluated on a sphere, the maxima of the resulting spherical function are indicative of the underlying tissue micro-structure.

We use the estimated 4th order diffusion tensor and extrapolate in q-space to synthetically simulate diffusion signal on a lattice in a box. Using this synthetic signal we numerically compute the Fourier transform and from there evaluate the diffusion propagator on a sphere.

\section{RESULTS}

We tested our modified approach on three test cases. The first was a statistical test on a synthetic dataset with known fiber 
directions. The second was a phantom dataset with fibers crossing perpendicularly. And the third was a real human brain dataset within a selected region with crossing fibers. In each of the cases we estimated the diffusion tensors from the DWIs, computed the diffusion propagators, and extracted the maxima of the propagators evaluated on a sphere.

The synthetic dataset was generated using a multi-tensor model, with a 2nd order tensor to simulate one fiber direction. We used a configuration of two fibers crossing perpendicularly with known ground truth directions. The synthetic DWIs were generated for 81 directions with a b-value of 3000 $\mathrm{mm}^{2} / \mathrm{s}$. The signal was corrupted with a random Rician noise with SNR: 5, 10, 20, 30, 40, 50. For each SNR level we generated 100 test cases. We estimated the diffusion tensors using two algorithms. The first was a simple Least Squares (LS) without a non-negative diffusion constraint, and the second was our modified TQ approach. The extracted maxima were then compared to the known ground truth directions and the mean error in degrees and the variance were computed. The results are presented in Fig-1. This experiment confirms the importance of the non-negative diffusion constraint.

The phantom dataset [6] was acquired on a GE Healthcare Signa 1.5T scanner. It had 4000 gradient directions and for our experiments we used a b-value of $4000 \mathrm{~mm}^{2} / \mathrm{s}$. The phantom had a geometry of two fiber bundles crossing perpendicularly close to the $\mathrm{X}$-axis and the Y-axis. Fig.2 shows the diffusion propagators and their extracted maxima. The extracted maxima strongly indicate the known goemetry of the phantom. The average computation time on our $2 \mathrm{GHz}$ Intel dual processor was NxMx0.0021875 seconds, with $\mathrm{N}$ gradient directions and $\mathrm{M}$ voxels.

In case of the real human brain dataset [7], we selected a region of interest (ROI) where on the coronal slice three major fiber bundles are known to cross. The ROI contained fiber bundles from the cortico-spinal tract, superior longitudinal fibers (traversing the plane) and the corpus callosum (in the plane). It was acquired on a $3 \mathrm{~T}$ Siemens scanner, with 60 gradient directions and a b-value of $1000 \mathrm{~mm}^{2} / \mathrm{s}$. Fig-3 shows the diffusion propagators and the extracted maxima.

\section{CONCLUSION}

In D-MRI it is essential to accurately reconstruct a diffusion function which faithfully reflects the underlying tissuemicrostructure. Since diffusion is a physical quantity, it is important that the diffusion function be non-negative in all directions. The authors of [3] suggested a TQ approach to estimate a non-negative diffusion function described by a 4th order diffusion tensor. They parameterized the TQ using Hilbert's theorem. However in their approach they faced an ambiguity of an orthogonal matrix class due to their formulation.

We revisited their approach, and realizing that the unknowns that needed to be estimated from the DWIs were the

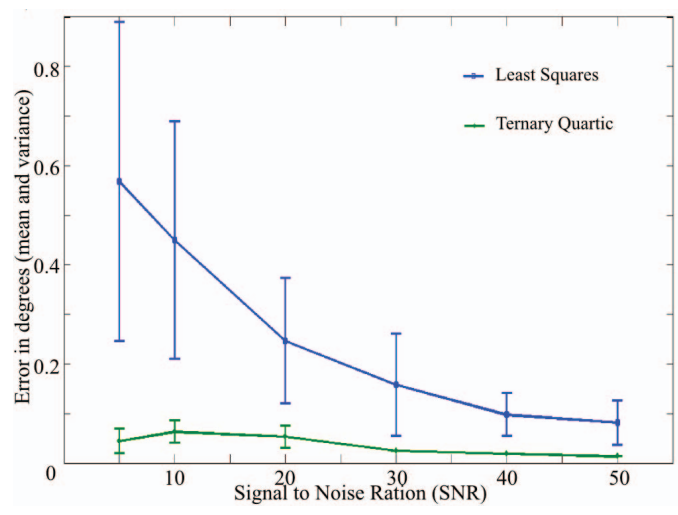

Fig. 1. Synthetic dataset with varying SNR. We compared the extracted maxima of the propagators to the known ground truth directions. This shows the importance of the nonnegative diffusion constraint.

coefficients of the quadratic forms we improved the above formulation with a simple modification. This naturally overcame the orthogonal matrix class ambiguity.

We applied our modified approach to synthetic and phantom data with known fiber configurations and also to a real human brain dataset. In all the cases after estimating the 4th order diffusion tensor we numerically computed the diffusion propagator using a Fourier transform. We extracted the maxima of the propagators evaluated on a sphere and were able to retrieve the dominant fiber directions. In the synthetic case, with known ground truth directions, we compared the TQ approach to the LS approach to show the importance of the non-negative diffusion constraint confirming previous results. And in the phantom case the extracted fiber directions corresponded strongly to the known geometry of the fibers.

However, we estimated 18 coefficients to calculate the Gram matrix from which we extracted the 15 independent coefficients of a symmetric 4th order tensor. We surmise that these extra coefficients can be resolved by introducing supplementary constraints to the problem

It is also interesting to note that using either of the approaches the estimated coefficients $\mathbf{C}$ and their negative values $-\mathbf{C}$ give rise to the same Gram matrix and therefore the same diffusion tensor. To overcome this the authors of the recently published paper [8] offer another novel parameterization of $\mathbf{C}$ with 6 additional constraints. This permits them to do a spatial regularization on $\|\nabla \mathbf{C}\|$ unambiguously. We are in the process of comparing our modified approach to this latest publication.

\section{REFERENCES}

[1] P.J. Basser, J. Mattiello, and D. LeBihan, "Estimation of the effective self-diffusion tensor from the NMR spin 


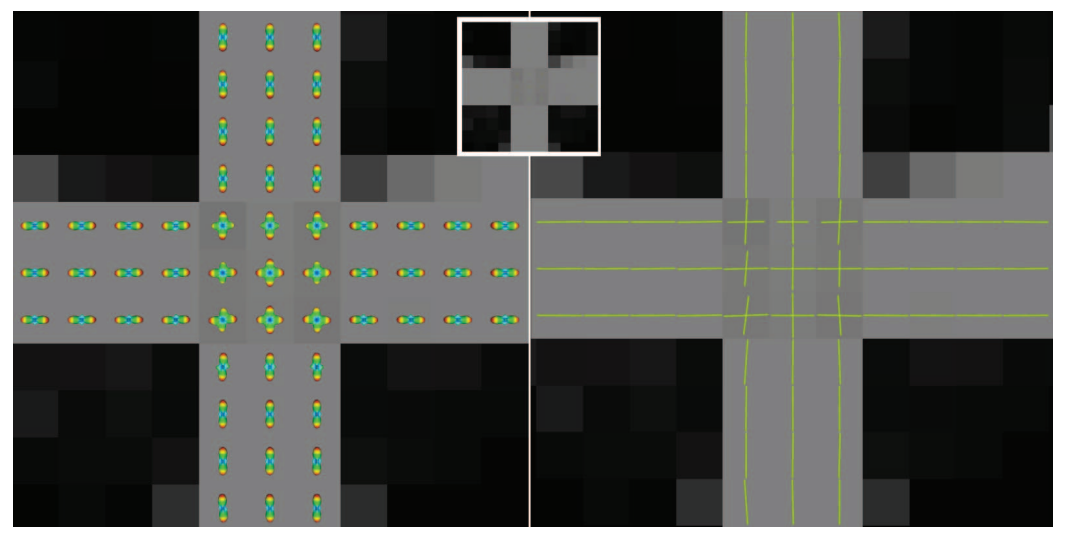

Fig. 2. Phantom dataset. The phantom had a geometry of two fiber bundles crossing perpendicularly close to the X-axis and the Y-axis. We estimated the 4th order diffusion tensor using our modified approach, from there computed the diffusion propagator, and its maxima when evaluated on a sphere. The extracted maxima strongly indicate the known fiber geometry of the phantom.

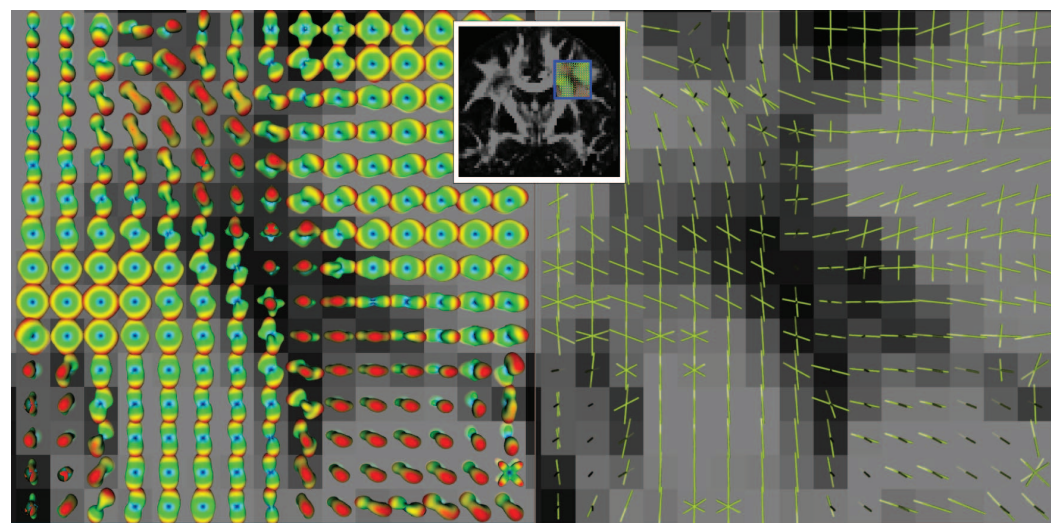

Fig. 3. Real human brain dataset. The ROI on a coronal slice contains fiber bundles from the cortico-spinal tract, superior longitudinal fibers (traversing the plane) and the corpus callosum (in the plane). We first estimated the 4th order diffusion tensor using our modified approach, from there computed the diffusion propagator, and its maxima when evaluated on a sphere.

echo," Journal of Magnetic Resonance, vol. B, no. 103, pp. 247-254, 1994.

[2] Evren Ozarslan and Thomas H Mareci, "Generalized diffusion tensor imaging and analytical relationships between diffusion tensor imaging and high angular resolution diffusion imaging.," Magn Reson Med, vol. 50, no. 5, pp. 955-965, Nov 2003.

[3] B. Jian B. C. Vemuri A. Barmpoutis and T. M. Shepherd, "Symmetric positive 4th order tensors \& their estimation from diffusion weighted mri," In LNCS 4584 (Springer) Proceedings of IPMI07: Information Processing in Medical Imaging, pp. 308-319, 2-6 July 2007.

[4] Victoria Powers and Bruce Reznick, "Notes towards a constructive proof of hilbert's theorem on ternary quartics," Quadratic forms and their applications (Dublin 1999), Contemp. Math., vol. 272, no. 9, 2000.
[5] E. Ozarslan, T.M. Shepherd, B.C. Vemuri, S.J. Blackband, and T.H. Mareci, "Resolution of complex tissue microarchitecture using the diffusion orientation transform (dot)," Tech. Rep. TR05-004, University of Florida, July 2005.

[6] C. Poupon, B. Rieul, I. Kezele, M. Perrin, F. Poupon, and J.-F. Mangin, "New diffusion phantoms dedicated to the study and validation of hardi models," Magnetic Resonance in Medicine, vol. 60, no. 6, pp. 1276-83, 2008.

[7] A. Anwander, M. Tittgemeyer, D. Y. von Cramon, A. D. Friederici, and T. R. Knosche, "Connectivity-based parcellation of broca's area," Cerebral Cortex, vol. 17, no. 4, pp. 816-825, 2007.

[8] M. S. Hwang D. Howland J. R. Forder A. Barmpoutis and B. C. Vemuri, "Regularized positive-definite fourth-order tensor field estimation from DW-MRI," NeuroImage, vol. 45, no. 1 sup.1, pp. 153-162, March 2009. 